\title{
Early osseointegration driven by the surface chemistry and wettability of dental implants
}

\begin{abstract}
Suelen Cristina SARTORETTO ${ }^{1}$, Adriana Terezinha Neves Novellino ALVES ${ }^{1}$, Rodrigo Figueiredo Britto RESENDE José CALASANS-MAIA², José Mauro GRANJEIRO ${ }^{3,4}$, Mônica Diuana CALASANS-MAIA ${ }^{4}$
\end{abstract}

\author{
1- Graduate Program in Dentistry, Fluminense Federal University, Niteroi, RJ, Brazil. \\ 2- Orthodontics Department, Fluminense Federal University, Niteroi, RJ, Brazil. \\ 3- National Institute of Metrology, Quality and Technology, Duque de Caxias, RJ, Brazil. \\ 4- Dental Clinical Research Center, Dentistry School, Fluminense Federal University, Niteroi, Rio de Janeiro, Brazil.
}

Corresponding address: Suelen Cristina Sartoretto - Rua Mario Santos Braga, 28/3andar - Center - Niteroi - RJ - Brazil - 24020-140 - Phone: +55 (21) 2629-9803 - Fax: +55 (21) 2629-9803 - e-mail: susartoretto@hotmail.com

Submitted: November 24, 2014 - Modification: March 29, 2015 - Accepted: April 6, 2015

\section{ABSTRACT}

\begin{abstract}
$\mathrm{O}$ bjective: The objective of this study was to investigate the impact of two different commercially available dental implants on osseointegration. The surfaces were: sandblasting and acid etching (Group 1) and sandblasting and acid etching, then maintained in an isotonic solution of $0.9 \%$ sodium chloride (Group 2). Material and Methods: X-ray photoelectron spectroscopy (XPS) was employed for surface chemistry analysis. Surface morphology and topography was investigated by scanning electron microscopy (SEM) and confocal microscopy (CM), respectively. Contact angle analysis (CAA) was employed for wetting evaluation. Bone-implant-contact (BIC) and bone area fraction occupied (BAFO) analysis were performed on thin sections $(30 \mu \mathrm{m}) 14$ and 28 days after the installation of 10 implants from each group $(n=20)$ in rabbits' tibias. Statistical analysis was performed by ANOVA at the $95 \%$ level of significance considering implantation time and implant surface as independent variables. Results: Group 2 showed 3-fold less carbon on the surface and a markedly enhanced hydrophilicity compared to Group 1 but a similar surface roughness $(p>0.05)$. BIC and BAFO levels in Group 2 at 14 days were similar to those in Group 1 at 28 days. After 28 days of installation, BIC and BAFO measurements of Group 2 were approximately 1.5 -fold greater than in Group $1(\mathrm{p}<0.05)$. Conclusion: The surface chemistry and wettability implants of Group 2 accelerate osseointegration and increase the area of the bone-to-implant interface when compared to those of Group 1.
\end{abstract}

Keywords: Dental implants. Surface properties. Wettability. Osseointegration. Rabbits.

\section{INTRODUCTION}

Implants, when in contact with the biological environment, are characterized by dynamic changes in their surface properties. Interaction between the tissues and the implant surfaces began immediately after the placement of the biomaterial in the body, and has been a challenge for scientists to increase the implant's biocompatibility and osteoconductivity ${ }^{12}$. Also, it has been difficult to determine the optimal modification required to accelerate the biological events that lead to more rapid osseointegration ${ }^{6}$.

The cascade of reactions that occurs between the biological environment and the biomaterial, this leads to the formation of a biofilm, which modulates the host's cellular responses. This event involves the blood clotting and the implant, with a thin layer of serum protein, which progresses to the granulation tissue, followed by immature woven bone ${ }^{1}$. The bone formation begins early, during the first week, through the promotion of osteoblast differentiation, production of osteogenic factors, cytokines and growth factors ${ }^{27}$. The primary bone that includes trabecular of woven bone is substituted by parallelfibered and/or lamellar bone and marrow. Between 1 and 2 weeks, the bone tissue responsible for primary mechanical stability of the device, immediately lateral to the implant region, is resorbed and substituted by newly formed bone ${ }^{1}$.

Currently, the abbreviation of the oral rehabilitation 
time without compromising osseointegration is a topic of particular interest in dentistry ${ }^{27}$. It is important to consider that the human body needs a minimum amount of time to perform reactions that result in osseointegration ${ }^{8}$. Early osseointegration provides the basis for current treatment protocols, for example, the immediate loading of dental implants consists of implant loading until 1 week after implant placement and early loading of dental implants consists of functions after 1 week and until 2 months after placement ${ }^{26}$.

The surface properties of dental implants appear to be one of the parameters affecting the speed of osseointegration, becoming an interesting alternative to enhance the bone-to-implant interface and improve osseointegration ${ }^{29}$.

The modification of dental implant surfaces often involves physical and topographical features at the micro and nanoscale, such as coating, roughness, porosity, patterning and fractal architecture. Additionally, changes in the chemical composition at the surface by plasma ${ }^{21}$ treatment or by oxidization ${ }^{5}$ affects osseo integration. Among the properties exhibited by dental implant's surfaces, several manuscripts have presented the properties exhibited by dental implant surfaces affecting osseointegration, such as, morphology ${ }^{8}$, topography ${ }^{28}$, roughness ${ }^{16}$, chemical and surface composition ${ }^{22}$, surface energy ${ }^{30}$, and hydrophilicity (or wettability) ${ }^{25}$. Moreover, there is an interaction between multiple factors that might interfere with the stability of the implants and, consequently, in the maintenance of osseointegration. For example, the dimensions and the shape of the implant, bone quality, surgical procedures and the loading conditions ${ }^{8}$.

The implant surface energy is an important factor for regulating osteogenesis. Depending on the surface energy, a hydrophilic or hydrophobic surface could prevail ${ }^{18}$. Generally, when the implant surface is positively charged, the surface becomes hydrophilic, and some of the plasma proteins essential for establishing the initial osteogenic interactions adsorb to hydrophilic surfaces ${ }^{11}$.

Hydrophilicity is a property that has gained increasing interest as a factor that affects the osseointegration of dental implants ${ }^{17}$. Hydrophilicity presents major advantages during the initial stages of wound healing and during the cascade of events that occurs during osseointegration, facilitating bone integration ${ }^{4}$. Studies suggest that osteoblasts cultured in chemically pure and hydrophilic surfaces express higher levels of differentiation markers such as alkaline phosphatase and osteocalcin when compared to hydrophobic surfaces ${ }^{30}$. Additionally, animal ${ }^{4}$ and clinical studies ${ }^{12}$ support the positive effect of hydrophilicity on osseointegration by significant improvements in bone-implant contact (BIC) and bone anchorage during the early stages of bone healing.

Physical and chemical changes in the surfaces of dental implants enhance the surfaces' hydrophilicity. A hydrophilic implant exhibits a reduced carbon concentration, which results in an increase in the amount of oxygen on its surface ${ }^{24}$. Theoretically, an oxide surface is hydrophilic, and when it binds structurally to water, $-\mathrm{OH}$ and $-\mathrm{O}_{2}$ groups are formed on its outermost layer ${ }^{23}$. Another hypothesis is that the formation of a hydroxylated oxide surface enhances reactivity toward the surrounding ions, amino acids and proteins in the tissue fluid.

The global implant market show a number of similar products alleging to have the "best" physicochemical, biological and clinical properties ${ }^{4,12,13}$. However, the regulators and professionals must have evidence showing the safety and efficacy of them through standardized protocols, to reduce controversy and the use of inefficient products. Therefore, this study aimed to investigate the impact of the surface properties of new commercially available dental implants on osseointegration, through surface chemistry, morphology, topography, and wetting analysis and a histomorphometric evaluation of bone-implant-contact (BIC) and bone area fraction occupied (BAFO).

\section{MATERIAL AND METHODS}

Implants: Two groups of Grade 4 titanium dental implants, Titamax, morse taper junction CM, (Neodent, Curitiba, PR, Brazil) were evaluated: Neoporos (Group 1) and Acqua (Group 2). The materials were sterilized by gamma radiation according to ISO 11137 (Sterilization of health care - products - Radiation $)^{10}$.

Group 1 surface: sandblasting with abrasive particles followed by an acid etching process.

Group 2 surface: as treated in Group 1 and, also, maintained in an isotonic solution of $0.9 \%$ sodium chloride.

\section{Surface characterization}

Scanning electron microscopy (SEM): scanning electron microscopy SSX-550 (Shimadzu, Kyoto, Kyoto, Japan) enable a qualitative characterization of the surface morphology of Group 1 and Group 2 implants ( $n=2 /$ group) at an accelerating voltage of $15 \mathrm{kV}$, focal width (FW) of 4.0, and Working Distance (WD) of 18 and 19 for magnifications of $1000 \mathrm{X}$ and 3000X, respectively.

Confocal three-dimensional laser scanning microscopy: $\mu$ Surf Custom (Nanofocus AG, Oberhausen, North Rhine - Westphalia, Germany) allowed for the quantitative evaluation of the surface roughness of different implant surfaces. Three implants of each group were used to measure the surface roughness values (mean values). The $\mu$ Soft 
Analysis Premium software program (Nanofocus AG, Oberhausen, North Rhine - Westphalia, Germany) was used to calculate $3 \mathrm{D}$ roughness parameters such as Ssk (amplitude distribution skew), Sa (arithmetic mean deviation of the peak-to-valley height of the surface), Sz (the average distance between the highest peak and the deepest valley), $\mathrm{Sdr}$ (indicates the surface area enlargement) and Sds (indicates the density of peaks on the surface). The Ssk parameter identifies the distribution of valleys $(S s k<0)$ or peaks $(S s k>0)$ on the surface. If the Ssk is close to zero, it has a gaussian surface ${ }^{21,30}$.

\section{$\mathrm{X}$-ray photoelectron spectroscopy (XPS)}

The chemical composition of the different groups was examined by $\mathrm{X}$-ray photoelectron spectroscopy (XPS) VG/SSI 2803 S-Probe (Kratos Analytical Ltd., Hofheim, Hesse, Germany) using an aluminum anode at $300 \mathrm{~W}(15 \mathrm{kV} \times 20 \mathrm{~mA})$. A monochromatic Al $\mathrm{K}_{\mathrm{a}}$ radiation source was used; the analysis spot size was $0.25 \times 1 \mathrm{~mm}$, and the measurement step size was $1 \mathrm{eV}$. Overview spectra were obtained over an energy range of $0-1100 \mathrm{eV}$. The emission angle between the incident beam and the surface of the sample was $35^{\circ}$. For elemental analysis, the survey spectra were analyzed and the detected elements were normalized. Two disks of each group were used for analysis.

Contact angle analysis: Universal Goniometer DSA 20E (KrüssHamburg, Germany) allowed the static contact angles on disks to mimic the implant surfaces for the hydrophilicity analysis by assessing by sessile-drop technique ${ }^{18}$. The measurements were performed on the surfaces ( $n=5$ disks/group) after a drop of SBF (simulated body fluid) solution was deposited onto the sample surfaces at room temperature. Fifteen contact angle values were obtained (one value per second in each sample).

\section{In vivo procedures}

Ten skeletally mature White New Zealand rabbits (Oryctolaguscuniculus) aged 5-6 months with an average weight of $3.3 \mathrm{~kg}$ (range, 3.0 to $3.5 \mathrm{~kg}$ ) of both sexes were used in this study. The animals were randomly assigned to individual $80 \times 80 \times 80$ $\mathrm{cm}$ cages and received water and a commercial chow diet ad libitum. Animal experiments and breeding were performed under conditions that were approved by the Institutional Review Board (CEP/ UFF: 380/2013), in compliance with the NIH Guide for Care and Use of Laboratory Animals and with Brazilian legislation on animal use.

\section{Surgical procedures}

The animal's legs were shaved before surgery. For experimental manipulation, all animals were anesthetized with an intramuscular injection of ketamine, Francotar ${ }^{\circledR} 20 \mathrm{mg} / \mathrm{kg}^{-1}$ (Virbac, Jurubatuba, SP, Brazil) and xylazine, Sedazine ${ }^{\circledR} 1$
mg/Kg-1 (FortDodge, São Cristovão, RJ, Brazil) to decrease the vagal tone. After 10 minutes, with visibly sedated animals exhibiting no responses to pain, ear vein puncture was performed, and lactated Ringer's solution (Baxter Hospitalar LTDA, São Paulo, $\mathrm{SP}$, Brazil) was administered intravenously at $15 \mathrm{ml} /$ $\mathrm{kg} / \mathrm{h}$. Then, the general anesthetic was maintained by the inhalation of $1 \%$ Isoflurane ${ }^{\circledR}$ (Cristália, Rio de Janeiro, RJ, Brazil). All surgical areas were clipped, prepared and draped using a sterile technique. Following the trichotomy and disinfection of both tibia regions with a $0.2 \%$ chlorhexidine solution, Riohex Scrub ${ }^{\circledR}$ (Rioquimica, São José do Rio Preto, SP, Brazil), infiltration anesthesia was applied using 2\% lidocaine and 1:100,000 epinephrine, Alphacaine $^{\circledR} 0.5$ - $1 \mathrm{ml} / \mathrm{site}$ (DFL, Rio de Janeiro, $\mathrm{RJ}$, Brazil) to reduce stimulation during surgery and generate vasoconstriction.

A $2-\mathrm{cm}$ incision was made in the epithelium lining of both rabbits' tibias. The soft tissue and periosteum were removed, and a sharp subperiosteal dissection exposed the proximal tibia, one implant was placed per limb. Under constant irrigation with $0.9 \%$ sodium saline solution, one perforation was made with a surgical drill according to the manufacturer's instructions. All drilling procedures were conducted at $1200 \mathrm{rpm}$. Immediately after opening, one implant was installed in the proximal site of each tibia, Group 1 on the right tibia and Group 2 on the left tibia ( $n=10 / p e r$ group). The periosteum around the bone perforations was then placed back in position and then attached to the subcutaneous tissue using an interrupted suture. The skin was closed using continuous \#5.0 nylon sutures Ethicon ${ }^{\circledR}$ (Johnson \& Johnson Medical Ltd., Blue Ash, Ohio, United States). Postoperatively, the rabbits received a single dose of an antibiotic, Pentabiótico Veterinário ${ }^{\circledR}$ (Fort Dodge, São Paulo, SP, Brazil), and an anti-inflammatory Meloxicam ${ }^{\circledR} 0.3$ $\mathrm{mg} / \mathrm{kg}$ (Ourofino, São Paulo, SP, Brazil). Each rabbit was caged individually and received food and water.

\section{Histological processing}

After 14 and 28 days, the animals were euthanized (10 rabbits/time point) with an overdose of sodium pentobarbital ( $60 \mathrm{mg} / \mathrm{kg} \mathrm{IM}$ ). A section of each tibia containing the implant area and 0.5 $\mathrm{cm}$ of excess tissue on each side were collected. The non-demineralized specimens were fixed for 2 days in $70 \%$ alcohol and gradually dehydrated using a series of alcohol solutions ranging from 70 to $100 \%$ ethanol. Specimens were then clarified in xylol and embedded in methylmetacrylate according to the manufacturer's instructions. The tissues were sliced ( $~ 300 \mu \mathrm{m}$ in thickness) through the center of the implant along its long axis with an Isomet 2000 precision diamond saw (Buehler Ltd., Lake Bluff, Illinois, United States), glued to acrylic 
plates with an acrylate-based cement Techonovit 7000 VCL (Külzer, Wehrheim, Hesse, Germany), and allowed to set for $24 \mathrm{~h}$ prior to grinding and polishing. The sections were then reduced to a final thickness of $\sim 30 \mu \mathrm{m}$ by grinding/polishing using a series of abrasive papers EXACT $310 \mathrm{CP}$ series $(800,1000,1200$ and 2400) (EXACT Apparatebau, Norderstedt, Schleswig-Holstein, Germany) under water irrigation. The unstained sections were analyzed by polarized light microscopy Axioplan 2 (Zeiss, Jena, Thuringia, Germany); the sections were then stained with toluidine blue and submitted to an optical microscopy evaluation Nikon Eclipse E400 (Nikon, Tokyo, Kanto, Japan).

\section{Histomorphometric analysis}

In each histological slice, eight non-superimposing fields, corresponding to the implant/bone interface (four fields on each side of the implant), were captured by scanning at a $20 x$ magnification, and digital image analysis software (Image ${ }^{\circledR} \mathrm{V} .1 .45$; National Institutes of Health, Bethesda, MD, USA) was used to measure the bone-to-implant contact (BIC) and bone area fraction occupied (BAFO). The regions of bone-to-implant contact along the implant perimeter were subtracted from the total implant perimeter, and calculations were performed to determine the BIC. The areas occupied by bone were subtracted from the total area between the threads, and calculations were performed to determine the BAFO [adapted from Bonfante, et al. ${ }^{2}(2012)$ ]. Both results were reported as percentages.

\section{Statistical analysis}

Because all samples of the in vivo test passed a normality test (Kolmogorov-Smirnov test), statistical analyses were performed by two-way
ANOVA, and Tukey's post hoc test to compare the differences between different experimental periods in the same group. Additionally, Student's t-tests were performed to determine differences between different groups during the same experimental period. The values obtained by confocal threedimensional laser scanning microscopy were analyzed with non-parametric Wilcoxon test. A statistical significance was indicated by $p$-levels of less than $5 \%$.

\section{RESULTS}

\section{Surface characterization}

\section{Scanning electron microscopy (SEM)}

SEM micrographs of the implant's surface revealed the textured microstructure of the samples. No marked differences between the groups were identified, and, in fact, the surface morphology of Group 1 appeared to be very similar to Group 2 in both magnifications (Figure 1 ).

\section{Confocal laser scanning microscopy}

In this study, it can be stated that the distribution of peaks and valleys is symmetric, there is a Gaussian surface in both implants and no statistical differences between the groups were observed (Figure 2). The Sa parameter provides a good overview of the values of the height of the irregularities on the surface. Based on the mean values obtained for Group 1 (1.44 $\mu \mathrm{m} \pm 1.15)$ and Group 2 (1.26 $\mu \mathrm{m} \pm 0.17)$ implants, it can be said that the two have a similar surface roughness, but only with this parameter, it is not possible to describe the surface as a whole. The Sz parameter indicates the average distance between the highest peak and the deepest valley. In this case, due to the process of obtaining the topography by the same method, the Sz value

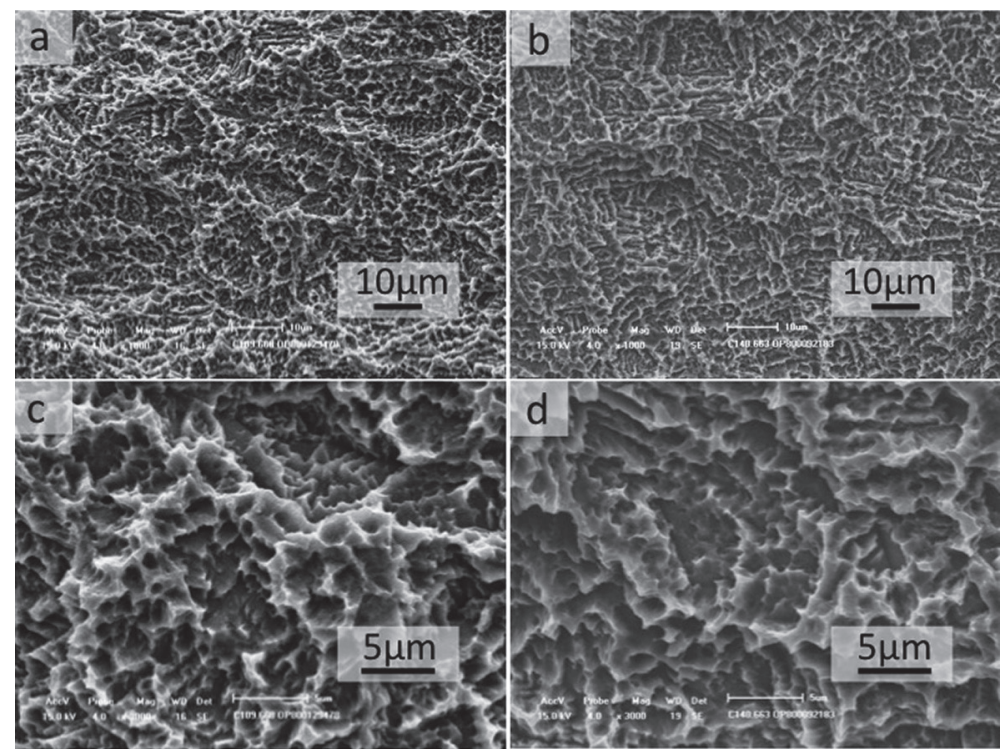

Figure 1- Scanning electron microscopy of implants surface. Group 1 (a and c) and Group 2 (b and d). No difference between the groups was observed. A and B (original magnification 1,000X) and C and D (original magnification 3,000X) 
doesn't change considerably (Group 1: $14.57 \mu \mathrm{m} \pm 1$ and Group 2: $16.20 \mu \mathrm{m} \pm 7.8)$. Sdr is the parameter that indicates the surface area enlargement. We can say that the surface area enlargement of Group 1 (mean value $1.51 \%$ ) is similar to the implant surface of Group 2 (mean value $1.21 \%$ ) due to the fact that the process of obtaining the topography is the same in both cases. The Sds parameter indicates the density of peaks on the surface. In this case, due to the process of obtaining the topography being the same, the Sds value does not change considerably when comparing both surface Group

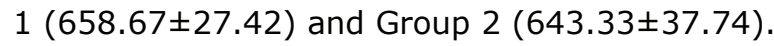

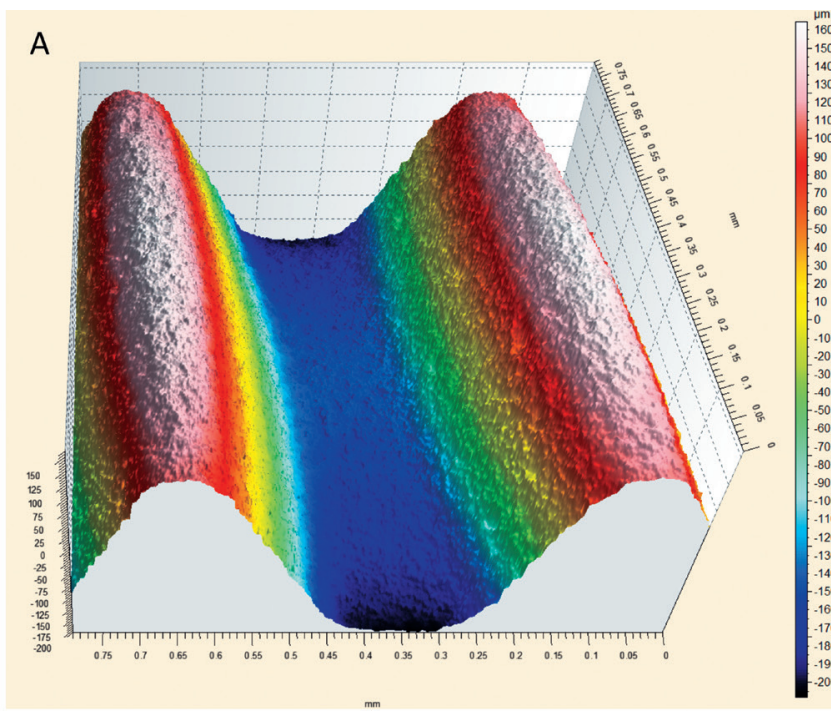

X-ray photoelectron spectroscopy (XPS)

XPS analysis showed peaks related to $\mathrm{C}, \mathrm{O}, \mathrm{N}$ and Ti for both groups tested. The surface of Group 1 presented atomic percentage values of 58 and 49 for carbon and 21 and 22 for oxygen, whereas Group 2 presented values of 17 for carbon and 59 for oxygen. Relative to the control surface, Group 2 showed a greater oxygen content.

\section{Contact angle analysis}

Group 2 presented superhydrophilic behavior (contact angle $<5^{\circ}$ ), whereas the surface of Group 1 specimens was hydrophobic (contact angle $>90^{\circ}$ ) (Table 1). The contact angles between the drop solution and different surfaces are shown in Figure 3.

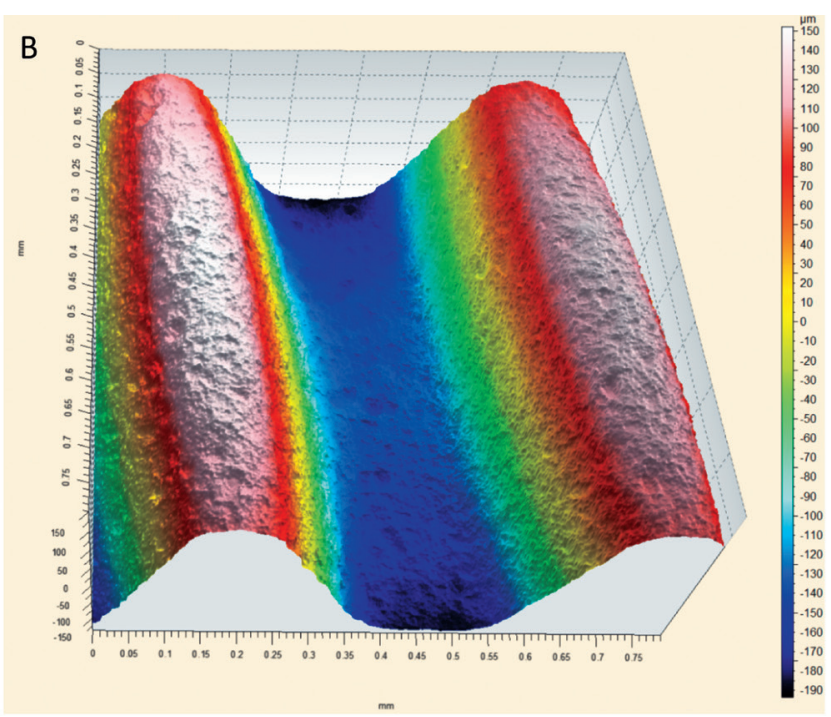

Figure 2- Confocal laser scanning microscopy in the thread region (a) Group 1 and (b) Group 2. No substantial difference between groups was observed (original magnification 20X). The numerical scale $(\mu \mathrm{m})$ from white $(160 \mu \mathrm{m})$ and black $(-200 \mu \mathrm{m})$

Table 1- Contact angle of different surfaces (goniometer)

\begin{tabular}{|c|c|c|c|c|c|c|c|}
\hline Surface & Disc $1\left(^{\circ}\right)$ & Disc $2\left(^{\circ}\right)$ & Disc $3\left(^{\circ}\right)$ & Disc $4\left(^{\circ}\right)$ & Disc $5\left(^{\circ}\right)$ & $\begin{array}{l}\text { Other } \\
\text { elements }\end{array}$ & $\begin{array}{l}\text { Standard } \\
\text { deviation }\end{array}$ \\
\hline Group 1 & 130.55 & 134.83 & 107.69 & 136.09 & 126.55 & 127.14 & 11.5 \\
\hline Group 2 & $<5^{\circ}$ & $<5^{\circ}$ & $<5^{\circ}$ & $<5^{\circ}$ & $<5^{\circ}$ & $<5^{\circ}$ & n.d. ${ }^{*}$ \\
\hline
\end{tabular}

* Not determinated

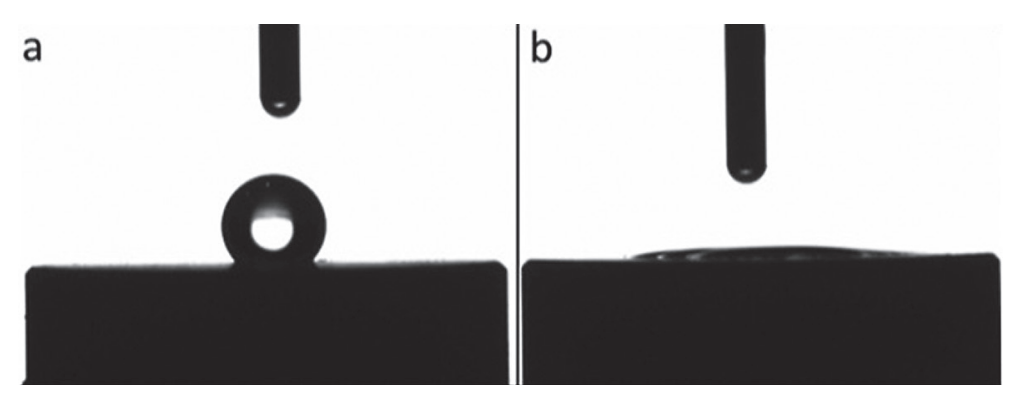

Figure 3- Contact angle of Group 1 (a) and Group 2 (b). It is possible see that a drop of simulated body fluid (SBF) solution had been deposited onto sample surfaces. Note that the angle formed between solution drop and Group 2 surface was smaller than $5^{\circ}$ 
In vivo evaluation

\section{Macroscopic evaluation}

All animals survived the experimental period, remained healthy and showed no discomfort. The surgical procedures and follow-ups presented no complications regarding procedural conditions, post-operative infection, or other clinical concerns. No implants were excluded from the study due to clinical instability immediately after euthanasia.

Histological analysis and histomorphometry

Non-decalcified, toluidine-blue-stained, thin sections allowed for the easy visualization of the
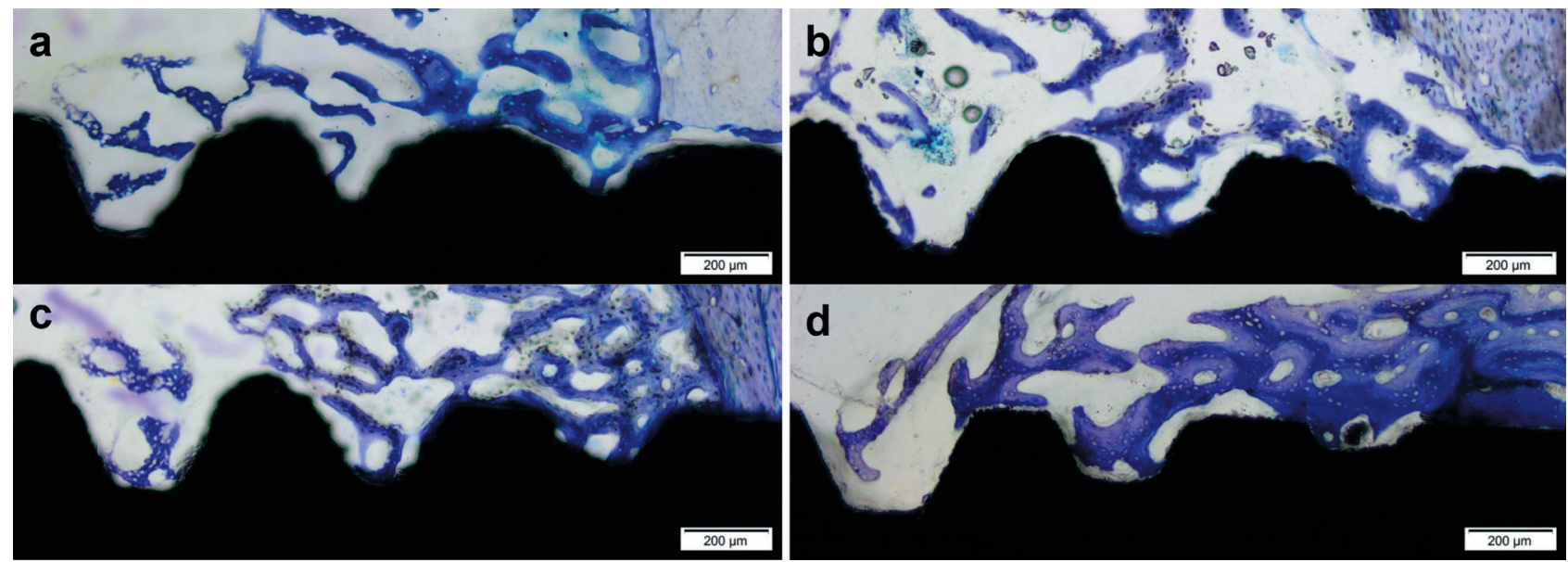

Figure 4- Photomicrographs of toluidine blue stained thin sections of the Group 1 at 14 days (a) and 28 days (b) and Group 2 at 14 days (c) and 28 days (d) in bright field (original magnification 10X). Observe the presence of new bone formation in the area between threads and the contact between bone and both implant groups. In Group 2 at 28 days, see the presence of trabecular bone more compact and in a greater number than Group 1 at the same period, suggesting the acceleration of osseointegration
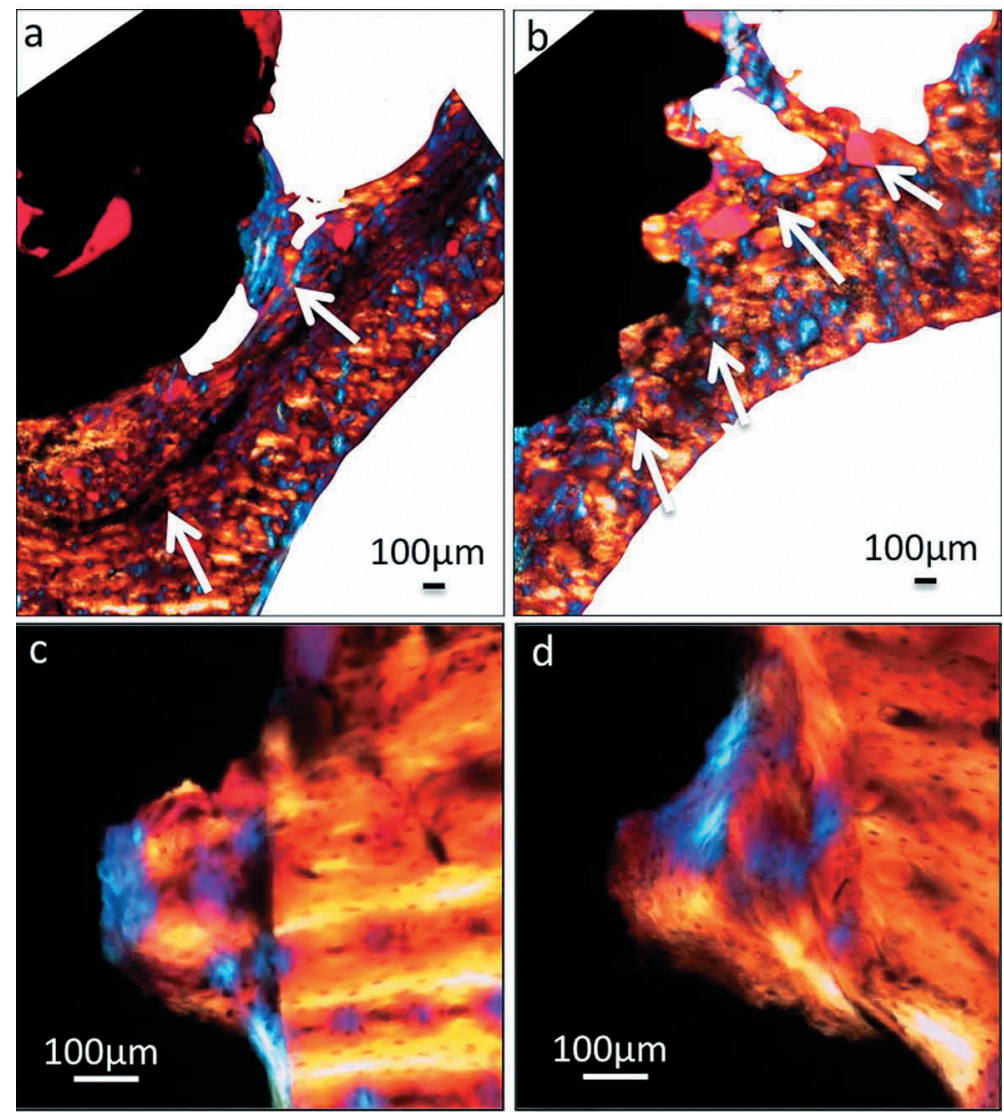

Figure 5- Polarized light micrographs of implant sections at the 28 day period. Apical region in Group 1 (a) and Group 2 (b) (original magnification 4X); cervical region in Group 1 (c) and Group 2 (d) (original magnification 20X). The arrows indicate the direction of osteoconduction from pre-existing bone in direction to the implant 

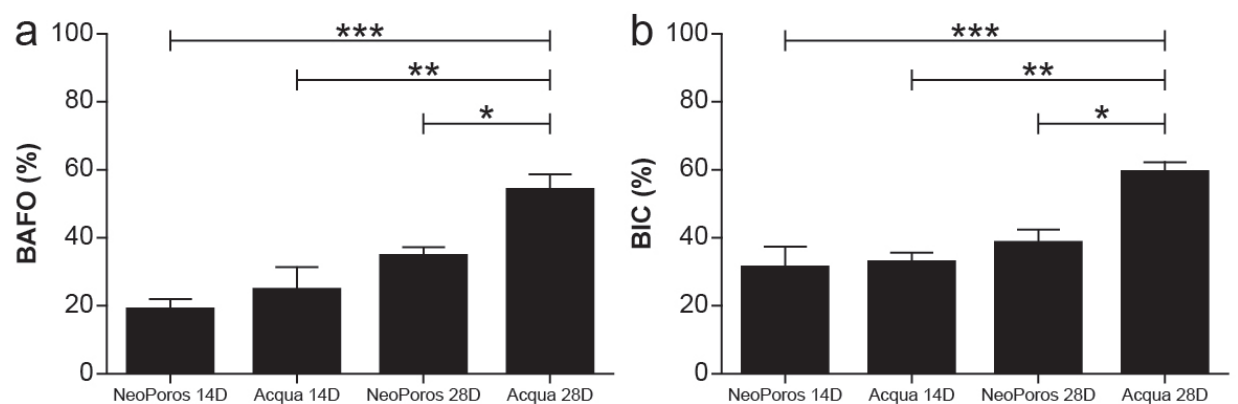

Figure 6- Histomorphometric analysis of (a) Bone area fraction occupied (BAFO) and (b) Mean bone-to-implant contact (BIC). BAFO was calculated as a percentage of the total region among the threads. BIC was calculated as a percentage of the total implant perimeter. Results are shown as mean percentages \pm standard deviation. Statistically significant differences are indicated by an asterisk, ${ }^{*} p<0.05 ;{ }^{* *} p<0.01 ;{ }^{* * *} p<0.001$

Table 2- Topographic analysis of the implant surface roughness, values obtained from confocal microscopy

\begin{tabular}{ccccccccc}
\hline Surface & Sample & SsK & $\begin{array}{c}\text { Sa } \\
{[\mu \mathrm{m}]}\end{array}$ & $\begin{array}{c}\text { Sz } \\
{[\mu \mathrm{m}]}\end{array}$ & Sdr & $\begin{array}{c}\text { Sda } \\
{\left[\mathrm{mm}^{2}\right]}\end{array}$ & $\begin{array}{c}\text { Sha } \\
{\left[\mathrm{mm}^{2}\right]}\end{array}$ & $\begin{array}{c}\text { Sds } \\
{\left[1 / \mathrm{mm}^{2}\right]}\end{array}$ \\
\hline Group 1 & 1 & -0.56 & 1.32 & 13.6 & 0.01 & 0 & 0 & 639 \\
& 2 & -0.55 & 1.39 & 14.5 & 0.01 & 0 & 0 & 647 \\
& 3 & -0.43 & 1.61 & 15.6 & 0.02 & 0 & 0 & 690 \\
& Mean & -0.51 & 1.44 & 14.57 & 0.02 & 0 & 0 & 658.67 \\
Group 2 & 1 & -0.3 & 1.26 & 11.4 & 0.01 & 0.29 & 0 & 669 \\
& 2 & -0.66 & 1.09 & 12 & 0.01 & 0 & 0 & 661 \\
& 3 & -0.33 & 1.43 & 25.2 & 0.01 & 0 & 0.01 & 600 \\
& Mean & -0.43 & 1.26 & 16.2 & 0.01 & 0.1 & 0.01 & 643.33 \\
\hline
\end{tabular}

bone-implant interfaces and determination of the bone-to-implant contact and bone area fraction occupied (Figure 4).

In both experimental periods, more islands of new trabecular bone between threads of the implants of Group 2 were observed than in the Group 1 (Figures 4A and 4D). Additionally, the new bone filled the region between the implant threads because the new bone was in intimate contact with the implant surface without the presence of an intervening fibrous tissue layer.

The histological findings obtained from polarized light microscopy showed greater osseoconduction for the new-formed bone in Group 2 in the apical region (Figures $5 \mathrm{~A}$ and $\mathrm{B}$ ) and also showed that the new bone in the area between threads was organized differently when compared with the lamellar structure of the pre-existing bone (Figures $5 C$ and D). Histological analysis was performed to further characterize the cervical and apical regions of the implants.

The results of histomorphometric analysis are summarized in Figure 6 and confirm the observations made by the histological analysis. Group 1 showed, after 28 days, rates of BIC of 38.6 $( \pm 8.76)$, while Group 2 presented $59.0( \pm 7.14)$ $(p<0.05)$ for the same parameter. For BAFO values,
Group1 presented $34.3( \pm 6.26)$ and Group 253.68 $( \pm 10.87)(p<0.05)$. It should be noted that the BIC and BAFO levels were similar between Group 2 at 14 days and Group 1 at 28 days ( $p>0.05$ ), clearly demonstrating an acceleration of osseointegration.

\section{DISCUSSION}

Over the past few years, material surface properties such as topography and wettability have received great attention in relation to dental implant applications ${ }^{4}$. An important modification is the improvement of the wettability of implant surfaces due to the biological implications of hydrophilicity, from the initial contact between an implant surface and host interface, which involves interactions with water and ions via conditioning by the formation of protein-rich films, up to the level of cellular interactions ${ }^{20}$. The general idea is that when wettability is increased, biocompatibility is enhanced, promoting interactions between an implant's surface and the biological environment, allowing for the activation of cellular activity most likely modulated by the implant surface's energy ${ }^{9}$.

Conventional surfaces are kept dry and exposed to air, making them hydrophobic due to the adsorption of carbon and the hydrocarbons present 
in the air and reducing the wetting of the implant by the surrounding biological environment; this process makes it difficult for proteins to be adsorbed and for cellular responses to be induced ${ }^{14}$. One of the principal strategies for preventing a decrease in the surface energy of titanium implants is the liquid isolation of the surface of $\mathrm{TiO}_{2}$ not contaminated by the atmosphere. Compared to conventional surfaces, these modified surfaces favor the adsorption of proteins and are able to promote the activation of osteoblasts toward a more osteogenic phenotype. These phenomena suggest that the increased bone formation observed on the modified surface of these implants should be due to the stimulating effect of high surface energy on osteoblasts ${ }^{30}$. Previous studies have shown that a hydrophilic surface is beneficial for gene expression, osteoblast behavior, bone mineralization and early osseointegration ${ }^{4,7}$.

Neither scanning electron microscopy (SEM) analysis nor confocal laser scanning microscopy revealed any remarkable differences in the morphological and topographical surfaces, respectively, between the two groups of implants. Similar results were obtained in another comparative study between two different surfaces, SLA and modSLA. SEM studies and three-dimensional topographical analysis by white-light confocal microscopy showed that the modified chemical surface (modSLA) had no structural impact when compared with the standard SLA, suggesting no topographical or morphological differences exist between SLA and modSLA. Indeed, no significant differences were observed for any of the surface roughness parameters ${ }^{4,15}$. In our study, the confocal microscopy showed that the distribution of peaks and valleys is symmetric between groups, both groups have a similar surface roughness (Sa, Sdr and Sds), the average distance between the highest peak and the deepest valley doesn't change considerably between groups (Sz) (Table 2).

In contrast, a significant difference in wettability, evaluated by contact angle analysis, was observed between the surfaces of groups, characterizing the surface of Group 2 as hydrophilic and Group 1 as hydrophobic $(p<0.05)$. These results corroborate those of two previous studies, one that compared two different implant surfaces, SLA and SLActive, in which the contact angle was evaluated and the authors demonstrated significant differences in wettability between the groups ${ }^{12}$, and the other compared four different types of surface modification with different wettability and nanostructures in a rabbit's tibia model and concluded that osseointegration was influenced by wettability, and super-hydrophilic surfaces demonstrated stronger bone response compared with hydrophobic surfaces in implants with the same microtopography ${ }^{29}$.

The greater amount of oxygen observed on
Group 2's surfaces can be attributed to the titanium oxide layer present on the surface of the implants ${ }^{24}$, in agreement with the results of other studies ${ }^{4,13}$. These values are similar to those reported in a previous study in which X-ray photoelectron spectroscopy analysis demonstrated that modSLA surfaces exhibited a higher concentration of oxygen $\left(\mathrm{O}_{2}, 55 \pm 2 \%\right)$ than their unmodified counterparts ${ }^{12}$. Conversely, modSLA surfaces yielded a lower carbon concentration (C, 18.4 $2.7 \%$ ) compared with standard SLA surfaces (C, 37.3 $\pm 3.4 \%$ ). The carbon dioxide concentration measured on the surface of Group 1's implants is also consistent with other results reported in studies that evaluated implant surfaces that had been exposed to air for a certain duration ${ }^{13}$.

The immersion of an implant in saline solution maintains the hydrophilicity of the implant surface, protecting it from air, preventing contamination by carbon and hydrocarbons and thus preventing the loss of surface energy ${ }^{24}$. This result is in agreement with the results reported in a study by Buser, et al. ${ }^{4}$ (2004) wherein the amount of carbon on the hydrophilic surface modSLA (control) implants placed in an isotonic solution of sodium chloride $(\mathrm{NaCl})$ decreased significantly compared to what had been observed on the conventional hydrophobic surface of SLA implants. This method for providing surface protection, in addition to maintaining hydrophilicity, also provides benefits such as exerting pro-osteogenic and pro-angiogenic effects ${ }^{7}$ and inducing a higher degree of osseointegration in both animals ${ }^{3}$, and humans ${ }^{12}$.

Higher surface hydrophilicity correlates positively with intense and rapid osteogenesis, as observed in this study. The results obtained in this study are in agreement with those of previous studies that demonstrated the beneficial properties of wettability in the early acceleration of osseointegration in both animals ${ }^{4}$ and humans ${ }^{12}$. Another important factor contributing to this improvement in the performance of Group 2 can be the support of the undisturbed preservation of blood clots on the "osteophilic" surface ${ }^{19}$.

In this study the in vivo results showed high rates of BIC and BAFO in Group 2 as compared with Group 1 after 28 days of implantation. This finding is in agreement with an animal study that showed significant improvements in BIC and bone anchorage during the early stages of bone healing in a minipig model. The authors showed an increase in BIC of $82 \%$ after 4 weeks for SLActive surface modification compared with the increase of $66.57 \%$ observed for the conventional SLA surface; thus, the improvement observed for the SLActive surface was 1.23-fold greater than that observed for the SLA surface. The BIC rates observed in minipigs after 28 days corresponded to the rates observed in this 
rabbit study after the same experimental period ${ }^{4}$. A clinical study also demonstrated an increase in the rate of BIC on SLActive implant surfaces (hydrophilic, $48.3 \%$ ) compared to that found on the SLA implant surfaces (hydrophobic, $32.4 \%$ ) $^{12}$.

The factors related to bone healing in our study were not affected by micro surface topography but by chemical changes.

\section{CONCLUSION}

This study demonstrated significant differences in the chemical properties and wettability between two groups of implants. Both surfaces had similar microtopography. The surface chemistry and wettability of Group 2's implants accelerated the implants' osseointegration and increased their boneto-implant interface relative to the findings obtained for Group 1's implants.

\section{REFERENCES}

1-Berglundh T, Abrahamsson I, Lang NP,Lindhe, J. De novo alveolar bone formation adjacent to endosseous implants. Clin Oral Implants Res. 2003; 14:251-62.

2-Bonfante EA, Witek L, Tovar N, Suzuki M, Marin C, Granato R, et al. Physicochemical characterization and in vivo evaluation of amorphous and partially crystalline calcium phosphate coatings fabricated on Ti-6Al-4V implants by the plasma spray method. Int J Biomater. 2012;2012:603826.

3-Brånemark PI, Adell R, Breine $U$, Hansson BO, Lindström J, Ohlsson A. Intra-osseous anchorage of dental prostheses. I. Experimental studies. Scand J Plast Reconstr Surg. 1969;3:81-100. 4-Buser D, Broggini N, Wieland M, Schenk RK, Denzer AJ, Cochran $\mathrm{DL}$, et al. Enhanced bone apposition to a chemically modified SLA titanium surface. J Dent Res. 2004;83:529-33.

5-Chiapasco M, Abati S, Romeo E, Vogel G. Clinical outcome of autogenous bone blocks or guided bone regeneration with e-PTFE membranes for the reconstruction of narrow edentulous ridges. Clin Oral Implants Res. 1999;10:278-88.

6-Coelho PG, Giro G, Teixeira HS, Marin C, Witek L, Thompson VP, et al. Argon-based atmospheric pressure plasma enhances early bone response to rough titanium surfaces. J Biomed Mater Res A. 2012;100:1901-6.

7-Donos N, Hamlet S, Lang NP, Salvi GE, Huynh-Ba G, Bosshardt $D D$, et al. Gene expression profile of osseointegration of a hydrophilic compared with a hydrophobic microrough implant surface. Clin Oral Implants Res. 2011;22:365-72.

8-Elias CN. Factors affecting the success of dental implants. In:Turkyilmaz I, ed. Implant dentistry: a rapidly evolving practice. Rijeka: InTech; 2011. p. 319-64.

9-Eriksson $\mathrm{C}$, Nygren $\mathrm{H}$, Ohlson K. Implantation of hydrophilic and hydrophobic titanium discs in rat tibia: cellular reactions on the surfaces during the first 3 weeks in bone. Biomaterials. 2004;25:4759-66.

10- International Organization for Standardization. ISO 11137: Sterilization of health care products - Radiation - Part 2: Establishing the sterilization dose. Geneva: ISO; 2013.

11-Jimbo R, Ivarsson M, Koskela A,Sul YT, Johansson CA. Protein adsorption to surface chemistry and crystal structure modification of titanium surfaces. J Oral Maxilofac Res. 2010;1:e3.

12-Lang NP, Salvi GE, Huynh-Ba G, Ivanovski S, Donos N, Bosshardt DD. Early osseointegration to hydrophilic and hydrophobic implant surfaces in humans. Clin Oral Implants Res. 2011;22:349-56.
13-Massaro C, Rotolo P, DeRiccardis F, Milella E, Napoli A, Wieland $M$, et al. Comparative investigation of the properties of commercial titanium dental implants. Part I: chemical composition. J Mater Sci Mater Med. 2002;13:535-48.

14-Michiardi A, Aparicio C, Ratner BD, Planell JA, Gil J. The influence of surface energy on competitive protein adsorption on oxidized NiTi surfaces.Biomaterials. 2007;28:586-92.

15-Quere D. Wetting and roughness. Annu Rev Mater Res. 2008;38:71-99.

16-Richards RG. The effect of surface roughness on fibroblast adhesion in vitro. Injury. 1996;27:SC38-43.

17-Rupp F, Scheideler L, Eichler M, Geis-Gerstorfer J. Wetting behavior of dental implants. Int J Oral Maxillofac Implants. 2011;26:1256-66.

18-Sawase T, Jimbo R, Baba K, Shibata Y, Ikeda T, Atsuta M. Photoinduced hydrophilicity enhances initial cell behavior and early bone apposition. Clin Oral Implants Res. 2008;19:491-6.

19-Schwarz F, Herten M, Sager M, Wieland M, Dard M, Becker J. Histological and immunohistochemical analysis of initial and early osseous integration at chemically modified and conventional SLA titanium implants: preliminary results of a pilot study in dogs. Clin Oral Implants Res. 2007;18:481-8.

20-Sela MN, Badihi L, Rosen G, Steinberg D, Kohavi D. Adsorption of human plasma proteins to modified titanium surfaces. Clin Oral Implants Res. 2007;18:630-8.

21- Shon WJ, Chung SH, Kim HK, Han GJ, Cho BH, Park YS. Periimplant bone formation of non-thermal atmospheric pressure plasma-treated zirconia implants with different surface roughness in rabbit tibiae. Clin Oral Implants Res. 2014;25:573-9.

22-Suzuki M, Guimarães MV, Marin C, Granato R, Gil JN, Coelho PG. Histomorphometric evaluation of alumina-blasted/acid-etched and thin ion beam-deposited bioceramic surfaces: an experimental study in dogs. J Oral Maxillofac Surg. 2009;67:602-7.

23-Tengvall P, Lundström I. Physico-chemical considerations of titanium as a biomaterial. Clin Mater. 1992;9:115-34.

24-Textor M, Sittig C, Frauchiger V, Tosatti S, Brunette DM. Properties and biological significance of natural oxide films on titanium and its alloys. In: Brunette DM, Tengvall $P$, Textor $M$, Thomsen $P$, eds. Titanium in medicine: material science, surface science, engineering, biological responses and medical applications. New York: Springer; 2001. p.171-230.

25-Vasak C, Busenlechner D, Schwarze UY, Leitner HF, Munoz Guzon F, Hefti T, et al. Early bone apposition to hydrophilic and hydrophobic titanium implant surfaces: a histologic and histomorphometric study in minipigs. Clin Oral Impl Res. 2014;25:1378-85.

26-Weber HP, Morton D, Gallucci GO, Roccuzzo M, Cordaro L, Grutter, L. Consensus statements and recommended clinical procedures regarding loading protocols. Int J Oral Maxillofac Implants. 2009;24:180-3.

27-Wennerberg A, Albrektsson T. Effects of titanium surface topography on bone integration: a systematic review. Clin Oral Implants Res. 2009;4:172-84.

28-Wennerberg A, Hallgren C, Johansson C, Danelli S. A histomorphometric evaluation of screw-shaped implants each prepared with two surface roughnesses. Clin Oral Implants Res. 1998.9:11-9.

29-Wennerberg A, Jimbo R, Stubinger S, Obrecht M, Dard M, Berner $S$. Nanostructures and hydrophilicity influence osseointegration: a biomechanical study in the rabbit tibia. Clin Oral Implants Res. 2014;25:1041-50.

30-Zhao G, Schwartz Z, Wieland M, Rupp F, Geis-Gerstorfer J, Cochran DL, et al. High surface energy enhances cell response to titanium substrate microstructure. J Biomed Mater Res A. $2005 ; 74: 49-58$ 